\title{
On Modular Standard Modules of Association Schemes
}

\author{
AKIHIDE HANAKI \\ hanaki@math.shinshu-u.ac.jp \\ Department of Mathematical Sciences, Faculty of Science, Shinshu University, \\ Matsumoto 390-8621, Japan \\ MASAYOSHI YOSHIKAWA \\ yoshi@math.shinshu-u.ac.jp \\ Department of Mathematical Sciences, Graduate School of Science and Technology, Shinshu University, \\ Matsumoto 390-8621, Japan
}

Received November 3, 2003; Revised June 24, 2004; Accepted July 1, 2004

\begin{abstract}
We will determine the structure of the modular standard modules of association schemes of class two. In the process, we will give the theoretical interpretation for the $p$-rank theory for strongly regular graphs, and understand the $p$-rank as the dimension of a submodule of the modular standard module. Considering the modular standard module, we can obtain the detailed classification more than the $p$-rank and the parameters.
\end{abstract}

Keywords: association scheme, modular adjacency algebra, modular standard module, $p$-rank, strongly regular graph

\section{Introduction}

There are many examples of nonisomorphic association schemes such that adjacency algebras over the complex number field have the same structure constants (intersection numbers). The standard module over the complex number field is completely determined by the structure constants. So their standard modules over the complex number field are also isomorphic. In this case, their modular adjacency algebras are also isomorphic to each other. But there exist cases where their modular standard modules are nonisomorphic. Here, the modular standard module means the standard module over a positive characteristic field. Therefore the structure of the modular standard module can play a role for the structure theory of the association schemes.

In this paper, we will determine the structure of the modular standard modules of association schemes of class 2 . We will use the $p$-rank theory of strongly regular graphs which are studied by Brouwer, van Eijl [5], and Peeters [8]. We will give the theoretical interpretation for the $p$-rank theory, and understand the $p$-rank as the dimension of a submodule of the standard module. Considering the standard module, we can obtain the detailed classification more than the $p$-rank and the parameters. 


\section{Definition and preliminaries}

In this section, we assume $\mathfrak{X}$ to be commutative. Let $\mathfrak{X}=\left(X,\left\{R_{i}\right\}_{i=0, \ldots, d}\right)$ be an association scheme, and $A_{i}(i=0, \ldots, d)$ be its adjacency matrices. Put $n=|X|$ and $v_{i}$ the valency of $A_{i}$. Let us denote the adjacency algebra of $\mathfrak{X}$ over a field $L$ by $L \mathfrak{X}$ and the standard module by $L X$.

Let $(K, R, F)$ be a splitting $p$-modular system for the adjacency algebra, and let $(\pi)$ be the maximal ideal of $R$. We denote the image of the canonical epimorphism $R \rightarrow F$ by $*$ (For details about a $p$-modular system, see [7]).

Suppose that $\mathfrak{X}$ is commutative. Then

$$
R \mathfrak{X} / \pi R \mathfrak{X} \cong F \mathfrak{X}
$$

and $\pi R \mathfrak{X} \subseteq J(R \mathfrak{X})$ [7, Theorem I.14.1], so idempotents of $F \mathfrak{X}$ are liftable to idempotents of $R \mathfrak{X}$ [7, Theorem I.14.2]. Consider the primitive idempotents decomposition of $1_{F \mathfrak{X}}$ in $F \mathfrak{X}:$

$$
1_{F \mathfrak{X}}=f_{0}+\cdots+f_{s} \in F \mathfrak{X}
$$

then we have the primitive idempotents decomposition of $1_{R \mathfrak{X}}$ in $R \mathfrak{X}$ :

$$
1_{R \mathfrak{X}}=e_{B_{0}}+\cdots e_{B_{s}} \in R \mathfrak{X}
$$

such that $e_{B_{i}}^{*}=f_{i}$. These decompositions yield the decomposition of algebras. We call this $e_{B_{i}}\left(e_{B_{i}}^{*}\right)$ a block idempotent of $R \mathfrak{X}(F \mathfrak{X})$, and we write $B_{i}=e_{B_{i}} R \mathfrak{X}$ and $B_{i}^{*}=e_{B_{i}}^{*} F \mathfrak{X}$.

Let $e_{0}, \ldots, e_{d}$ be the set of primitive idempotents in $K \mathfrak{X}$. Then there is a partition $\{0, \ldots, d\}=\bigcup_{j=0}^{s} T_{j}$ such that $e_{B_{i}}=\sum_{j \in T_{i}} e_{j}$. When $e_{j} \in T_{i}$, we say that $e_{j}$ belongs to the block $B_{i}$.

Let $\chi_{j}$ be the (one-dimensional) irreducible representation of $K \mathfrak{X}$ corresponding to $e_{j}$. Then $e_{j}$ belongs to $B_{i}$ if and only if $\chi_{j}\left(e_{B_{i}}\right)=1$. Since $B_{i}^{*}$ has the unique idempotent $e_{B_{i}}^{*}$, $B_{i}^{*} / J\left(B_{i}^{*}\right) \cong F$. If $\chi_{i}$ and $\chi_{j}$ belong to the same block, then $\chi_{i}^{*}=\chi_{j}^{*}$. So we have the following.

Lemma 1 Irreducible representations $\chi_{i}$ and $\chi_{j}$ of $K \mathfrak{X}$ belong to the same block if and only if $\chi_{i}\left(A_{r}\right) \equiv \chi_{j}\left(A_{r}\right)(\bmod (\pi))$ for all $r=0, \ldots, d$.

Lemma 2 The dimension of $B_{i}^{*}$ is equal to the number of $\chi_{j}$ belonging to $B_{i}$.

Proof: We have $\operatorname{dim}_{F} B_{i}^{*}=\operatorname{rank}_{R} B_{i}=\operatorname{dim}_{K} e_{B_{i}} K \mathfrak{X}$.

We consider a $(d+1) \times(d+1)$ matrix $P$ with the $(i, j)$-entry $\chi_{i}\left(A_{j}\right)$. We call $P$ the character table of $\mathfrak{X}$.

Proposition 3 The algebra $F \mathfrak{X}$ is semisimple if and only if $\operatorname{det} P \not \equiv 0(\bmod (\pi))$. 
Proof: If $F \mathfrak{X}$ is semisimple, then clearly we have det $P^{*} \neq 0$. If $F \mathfrak{X}$ is not semisimple, the $P^{*}$ contains at least two same rows, so $\operatorname{det} P^{*}=0$.

General sufficient criteria for adjacency algebras to be semisimple were obtained in [1] and [6].

Mapping each matrix $A_{r}$ to $v_{r}$ induces an algebra homomorphism from $K \mathfrak{X}$ to $K$. This representation is usually called the trivial representation of $K \mathfrak{X}$. We assume that $\chi_{0}$ is the trivial representation, and $\chi_{0}$ belongs to $B_{0}$. We call $B_{0}$ the principal block of $\mathfrak{X}$. Then we have the following.

Proposition 4 The dimension of the principal block $B_{0}^{*}$ is one if and only if $p \nmid n$. (This is also true for non-commutative association schemes.)

Proof: Put $J=\sum_{i=0}^{d} A_{i}$. Since $J A_{i}=A_{i} J=v_{i} J, J^{*}$ generates a one-dimensional ideal of $F \mathfrak{X}$, and the corresponding representation is trivial. We have $J^{2}=n J$. If $p \nmid n$, then $n^{-1} J$ is a central primitive idempotent, thus $B_{0}^{*}=J^{*} F \mathfrak{X}$ is one-dimensional. If $p \mid n$, then $J^{*}$ is a central nilpotent element in $B_{0}^{*}$, so it is in the Jacobson radical. In this case, $\operatorname{dim}_{F} B_{0}^{*}>1$.

\section{Contragradient modules}

Let $\mathfrak{X}=\left(X,\left\{R_{i}\right\}_{i=0, \ldots, d}\right)$ be an association scheme (not necessary commutative), and $F$ a field. For a right $F \mathfrak{X}$-module $V$, we put $\hat{V}:=\operatorname{Hom}_{F}(V, F)$. For $f \in \hat{V}, v \in V$, and $i \in\{0, \ldots, d\}$, we define the action of $F \mathfrak{X}$ to $\hat{V}$ by

$$
\left(f A_{i}\right)(v):=f\left(v A_{i^{\prime}}\right)
$$

Here $A_{i^{\prime}}={ }^{t} A_{i}$. Then

$$
\left(f\left(A_{i} A_{j}\right)\right)(v)=f\left(v A_{j^{\prime}} A_{i^{\prime}}\right)=\left(\left(f A_{i}\right) A_{j}\right)(v) \quad \text { for } i, j \in\{0, \ldots, d\} .
$$

So $\hat{V}$ is a right $F \mathfrak{X}$-module. We call $\hat{V}$ the contragradient module of $V$.

Proposition $5 \widehat{F X} \cong F X$ as right $F \mathfrak{X}$-modules.

Proof: For $x \in X$, define $f_{x} \in \widehat{F X}$ by $f_{x}(y)=\delta_{x y}$. Put $\varphi: F X \rightarrow \widehat{F X}$ the $F$-linear map defined by $\varphi(x)=f_{x}$. Obviously $\varphi$ is an isomorphism of vector spaces. We show that 
$\varphi$ is an $F \mathfrak{X}$-homomorphism. We have

$$
\begin{aligned}
\varphi\left(x A_{i}\right)(y) & =\varphi\left(\sum_{(x, z) \in R_{i}} z\right)(y)=\sum_{(x, z) \in R_{i}} f_{z}(y) \\
& = \begin{cases}1 & \text { if }(x, y) \in R_{i}, \\
0 & \text { otherwise, }\end{cases} \\
\left(\varphi(x) A_{i}\right)(y) & =\varphi(x)\left(y A_{i^{\prime}}\right)=f_{x}\left(\sum_{(y, z) \in R_{i^{\prime}}} z\right) \\
& = \begin{cases}1 & \text { if }(y, x) \in R_{i^{\prime}}, \\
0 & \text { otherwise. }\end{cases}
\end{aligned}
$$

Now $\varphi\left(x A_{i}\right)=\varphi(x) A_{i}$ and $\varphi$ is an $F \mathfrak{X}$-homomorphism.

Proposition 6 We fix $x \in X$.Put $\iota: F \mathfrak{X} \rightarrow F X$ the $F$-linear map defined by $\iota\left(A_{i}\right)=x A_{i}$. Then $\iota$ is an $F \mathfrak{X}$-monomorphism.

Proof: Obviously, $\iota$ is an $F \mathfrak{X}$-homomorphism. Assume $\iota\left(\sum_{i=0}^{d} a_{i} A_{i}\right)=0$. Then

$$
0=\iota\left(\sum_{i=0}^{d} a_{i} A_{i}\right)=\sum_{i=0}^{d} a_{i} x A_{i}=\sum_{i=0}^{d} \sum_{(x, y) \in R_{i}} a_{i} y .
$$

Now $a_{i}=0$ for all $i \in\{0, \ldots, d\}$, and so $\sum_{i=0}^{d} a_{i} A_{i}=0$.

We consider the structure of the contragradient module $\widehat{F \mathfrak{X}}$ of the regular $F \mathfrak{X}$-module. Define $\tau_{i} \in \widehat{F \mathfrak{X}}$ by $\tau_{i}\left(A_{j}\right)=\delta_{i j}$. Then $\left\{\tau_{i} \mid i \in\{0, \ldots, d\}\right\}$ is a basis of $\widehat{F \mathfrak{X}}$. Now

$$
\left(\tau_{i} A_{j}\right)\left(A_{k}\right)=\tau_{i}\left(A_{k} A_{j^{\prime}}\right)=p_{k j^{\prime}}^{i}
$$

So we have the following.

Proposition $7 \quad \tau_{i} A_{j}=\sum_{k \in 0}^{d} p_{k j^{\prime}}^{i} \tau_{k}$.

Proposition 8 Let $\iota: F \mathfrak{X} \rightarrow F X$ be as above. Put $\hat{\imath}: \widehat{F X} \rightarrow \widehat{F \mathfrak{X}}$ the map defined by $\hat{\imath}(f)=f \circ \iota$. Then $\hat{\imath}$ is a F $\mathfrak{X}$-epimorphism.

Proof: For $i \in\{0, \ldots, d\}$, we fix $y \in X$ such that $(x, y) \in R_{i}$. Then

$$
\begin{aligned}
\hat{\imath}\left(f_{y}\right)\left(A_{j}\right) & =f_{y} \circ \iota\left(A_{j}\right)=f_{y}\left(x A_{j}\right) \\
& = \begin{cases}1 & \text { if }(x, y) \in R_{j}, \\
0 & \text { otherwise },\end{cases}
\end{aligned}
$$

and so we have $\hat{\imath}\left(f_{y}\right)=\tau_{i}$. 


\section{The structure of the adjacency algebras with $d=2$}

It is well-known that association schemes with at most five elements are commutative [12, Theorem 4.5.1(ii)]. We assume that $F$ is an algebraically closed field of characteristic $p$.

Lemma 9 The isomorphism classes of $F$-algebras of dimension 3 are $F \oplus F \oplus F$, $F \oplus F[x] /\left(x^{2}\right), F[x] /\left(x^{3}\right), F[x, y] /\left(x^{2}, x y, y^{2}\right)$, and $T_{2}(F)=\left(\begin{array}{cc}F & 0 \\ F\end{array}\right)$.

Since $T_{2}(F)$ is non-commutative, $T_{2}(F)$ can not be an adjacency algebra of an association scheme. The algebra $F[x, y] /\left(x^{2}, x y, y^{2}\right)$ is not self injective, but it can be an adjacency algebra of an association scheme. The smallest such example is the adjacency algebra of the group association scheme of the symmetric group of degree 3 in characteristic 3 .

Firstly, we consider the non-symmetric case, namely $A_{1^{\prime}}={ }^{t} A_{1}=A_{2}$. Put $k=v_{1}=$ $v_{2}=(n-1) / 2$, where $n=|X|$. Note that $k \equiv 3(\bmod 4)$, in this case. Then we have

$$
\begin{aligned}
A_{1}^{2} & =\frac{k-1}{2} A_{1}+\frac{k+1}{2} A_{2}, \\
A_{1} A_{2} & =k A_{0}+\frac{k-1}{2} A_{1}+\frac{k-1}{2} A_{2}, \\
A_{2}^{2} & =\frac{k+1}{2} A_{1}+\frac{k-1}{2} A_{2} .
\end{aligned}
$$

Theorem 10 Let $\mathfrak{X}$ be a non-symmetric association scheme with $d=2$. Then we have the following.

(1) If $p \nmid n$, then $F \mathfrak{X} \cong F \oplus F \oplus F$.

(2) If $p \mid n$, then $F \mathfrak{X} \cong F[x] /\left(x^{3}\right)$.

Proof: The character table is as follows:

\begin{tabular}{cccc}
\hline & $A_{0}$ & $A_{1}$ & $A_{2}$ \\
\hline$\chi_{0}$ & 1 & $k$ & $k$ \\
$\chi_{1}$ & 1 & $\frac{-1+\sqrt{n} i}{2}$ & $\frac{-1-\sqrt{n} i}{2}$ \\
$\chi_{2}$ & 1 & $\frac{-1-\sqrt{n} i}{2}$ & $\frac{-1+\sqrt{n} i}{2}$ \\
\hline
\end{tabular}

Obviously, (1) holds. (2) In this case, $\left(A_{1}^{*}-k A_{0}^{*}\right)^{2} \neq 0$ and $\left(A_{1}^{*}-k A_{0}^{*}\right)^{3}=0$, so we have $F \mathfrak{X} \cong F[x] /\left(x^{3}\right)$.

Now we consider symmetric association schemes with $d=2$. They correspond to strongly regular graphs. If we consider a strongly regular graph with parameters $(n, k, \lambda, \mu)$ $[4$, p. 8], then we have

$$
\begin{aligned}
A_{1}^{2} & =k A_{0}+\lambda A_{1}+\mu A_{2}, \\
A_{1} A_{2} & =(k-\lambda-1) A_{1}+(k-\mu) A_{2}, \\
A_{2}^{2} & =(n-k-1) A_{0}+(n-2 k+\lambda) A_{1}+(n-2 k+\mu-2) A_{2} .
\end{aligned}
$$


Now $A_{1}$ has three eigenvalues $k, r$, and $s$, here $r$ and $s$ are roots of the equation $x^{2}+$ $(\mu-\lambda) x+(\mu-k)=0$. The character table is as follows.

\begin{tabular}{cccc}
\hline & $A_{0}$ & $A_{1}$ & $A_{2}$ \\
\hline$\chi_{0}$ & 1 & $k$ & $n-k-1$ \\
$\chi_{1}$ & 1 & $r$ & $-r-1$ \\
$\chi_{2}$ & 1 & $s$ & $-s-1$ \\
\hline
\end{tabular}

If the graph is not a conference graph, then $r$ and $s$ are rational integers.

Theorem 11 Let $\Gamma$ be a strongly regular graph with parameters $(n, k, \lambda, \mu)$ which is not a conference graph, and let $\mathfrak{X}$ be an association scheme defined by $\Gamma$. Then $A_{1}$ has eigenvalues $k, r$, and $s$ and the following holds.

(1) If $p \mid n$ and $r \not \equiv s(\bmod p)$, then $F \mathfrak{X} \cong F[x] /\left(x^{2}\right) \oplus F\left(F[x] /\left(x^{2}\right)\right.$ is the principal block),

(2) If $p \mid n, r \equiv s(\bmod p)$, and at least one of $k(k+1), \lambda-2 k$ and $\mu$ are not zero $(\bmod$ $p)$, then $F \mathfrak{X} \cong F[x] /\left(x^{3}\right)$,

(3) If $p \mid n, r \equiv s(\bmod p)$, and $k(k+1) \equiv \lambda-2 k \equiv \mu \equiv 0(\bmod p)$, then $F \mathfrak{X} \cong$ $F[x, y] /\left(x^{2}, x y, y^{2}\right)$,

(4) If $p \nmid n$ and $r \not \equiv s(\bmod p)$, then $F \mathfrak{X} \cong F \oplus F \oplus F$,

(5) If $p \nmid n$ and $r \equiv s(\bmod p)$, then $F \mathfrak{X} \cong F \oplus F[x] /\left(x^{2}\right)$ ( $F$ is the principal block).

In (2) and $(3), k(k+1) \equiv \lambda-2 k \equiv \mu \equiv 0(\bmod p)$ if and only if $\left(A_{1}^{*}-k A_{0}^{*}\right)^{2}=0$.

Proof: We note that $r \equiv s(\bmod (\pi))$ if and only if $r \equiv s(\bmod p)$ since $r$ and $s$ are rational integers.

Suppose that $p \mid n$. Then the principal block is not simple, so $k \equiv r(\bmod p)$ of $k \equiv s$ $(\bmod p)$ by the character table. If only one of them holds, then (1) holds. Otherwise, $F \mathfrak{X}$ has only one irreducible representation, so it is a local algebra. In this case, we have $F \mathfrak{X} \cong F[x] /\left(x^{3}\right)$ or $F \mathfrak{X} \cong F[x, y] /\left(x^{2}, x y, y^{2}\right)$. If $\left(A_{1}^{*}-k A_{0}^{*}\right)^{2} \neq 0$, then we have $F \mathfrak{X} \cong F[x] /\left(x^{3}\right)$. Suppose $\left(A_{1}^{*}-k A_{0}^{*}\right)^{2}=0$. Then, by a direct calculation, we have $k(k+1) \equiv \lambda-2 k \equiv \mu \equiv 0(\bmod p)$ and $\left(\alpha A_{1}^{*}+\beta A_{2}^{*}\right)^{2}=0$ for any $\alpha, \beta \in F$. Thus $F \mathfrak{X} \cong F[x, y] /\left(x^{2}, x y, y^{2}\right)$.

Suppose that $p \nmid n$. Then the principal block is isomorphic to $F$. The result follows from Lemma 2.

Note that there exist strongly regular graphs for every case in this theorem.

Now we consider conference graphs. Then the parameters are $(n, k, \lambda, \mu)=(4 \mu+$ $1,2 \mu, \mu-1, \mu)$, and the eigenvalues are $k, r=(-1+\sqrt{n}) / 2$, and $s=(-1-\sqrt{n}) / 2$.

Theorem 12 Let $\Gamma$ be a conference graph with parameters $(n, k, \lambda, \mu)=(4 \mu+1,2 \mu, \mu-$ $1, \mu)$, and let $\mathfrak{X}$ be an association scheme defined by $\Gamma$. Then the following holds.

(1) If $p \mid n$, then $F \mathfrak{X} \cong F[x] /\left(x^{3}\right)$.

(2) If $p \nmid n$, then $F \mathfrak{X} \cong F \oplus F \oplus F$. 
Proof: Suppose $p \mid n$. Then $k \equiv r \equiv s(\bmod (\pi))$, so $F \mathfrak{X}$ is not semisimple. We can easily check that $\left(A_{1}^{*}-k A_{0}^{*}\right)^{2} \neq 0$, thus $F \mathfrak{X} \cong F[x] /\left(x^{3}\right)$.

Suppose $p \nmid n$. Then $r-s=\sqrt{n} \not \equiv 0(\bmod (\pi))$. Thus $F \mathfrak{X}$ is semisimple.

\section{The structure of the standard module with $d=2$}

In the previous section, we showed that there exist the following five types for the structure of the adjacency algebras:

(1) $F \mathfrak{X} \cong F \oplus F \oplus F$,

(2) $F \mathfrak{X} \cong F \oplus F[x] /\left(x^{2}\right)$ (the principal block is $F$ ),

(3) $F \mathfrak{X} \cong F[x] /\left(x^{2}\right) \oplus F$ (the principal block is $F[x] /\left(x^{2}\right)$ ),

(4) $F \mathfrak{X} \cong F[x] /\left(x^{3}\right)$,

(5) $F \mathfrak{X} \cong F[x, y] /\left(x^{2}, x y, y^{2}\right)$.

In this section, we will determine the structure of the standard module for each type. We assume that $A_{1}$ has eigenvalues $k, r, s$ with multiplicities $1, m_{r}, m_{s}$.

If $\mathfrak{X}$ is commutative, $(F X) M$ is an $F \mathfrak{X}$-module for any $M \in F \mathfrak{X}$. Then it follows that $\operatorname{dim}(F X) M=r k_{F}(M)$. Especially, if $\mathfrak{X}$ is class 2, the $p$-rank of $M$ such that $M \in \operatorname{rad} F \mathfrak{X}$ is very important. Since many of them have the relevant $p$-rank, the structure of $F X$ plays a role for the structure theory of the association scheme (See [5]).

\subsection{Type (1) $F \mathfrak{X} \cong F \oplus F \oplus F$}

Let $B_{0}, B_{1}$ and $B_{2}$ be the corresponding blocks to $k, r$ and $s$, respectively. Let $S_{i}$ be the simple module such that the corresponding representation belongs to $B_{i}$ for $i=0,1,2$. In this case, it is known that

$$
F X \cong S_{0} \oplus m_{r} S_{1} \oplus m_{s} S_{2} .
$$

In this case, the structure of $F X$ is completely determined by the parameters.

In the case of type (2) or type (3), we set that $F \mathfrak{X}=B_{0} \oplus B_{1}$ is the block decomposition of the adjacency algebra, where $B_{0}$ is the principal block. Let $S_{i}$ be the simple module such that the corresponding representation belongs to $B_{i}$ for $i=0,1$. In these cases, it is enough that we determine $\operatorname{dim}_{F} \operatorname{rad} F X$.

\subsection{Type (2) $F \mathfrak{X} \cong F \oplus F[x] /\left(x^{2}\right)$}

Let $P$ be the projective cover of $S_{1}$ and $e_{1}$ the block idempotent of $B_{1}$. We set that $M=$ $e_{1}\left(A_{1}-s A_{0}\right)$. Then $\operatorname{rad} F \mathfrak{X}=F M^{*}$. Since the coefficient of $A_{i}^{*}$ in $M^{*}$ is in $\mathbb{F}_{p}$ for each $i$, $r k_{F}(M)=r k_{p}(M)$. We set that $t=r k_{p}(M)$. Then it follows that

$$
F X \cong S_{0} \oplus t P \oplus(n-1-2 t) S_{1} .
$$


Interesting examples are the association schemes defined by $(26,10,3,4)$-strongly regular graphs. In this case, there are 10 non-isomorphic association schemes. The adjacency algebras over a field of characteristic 5 are type (2). Then the structures of the modular standard modules are as follows:

\begin{tabular}{lc}
\hline$F X$ & $\begin{array}{c}\text { The number of non-isomorphic } \\
\text { association schemes }\end{array}$ \\
\hline$S_{0} \oplus 9 P \oplus 7 S_{1}$ & 1 \\
$S_{0} \oplus 11 P \oplus 3 S_{1}$ & 2 \\
$S_{0} \oplus 12 P \oplus S_{1}$ & 7 \\
\hline
\end{tabular}

\subsection{Type (3) $F \mathfrak{X} \cong F[x] /\left(x^{2}\right) \oplus F$}

Let $P$ be the projective cover of $S_{0}$. In this case, we have either $k \equiv r$ or $k \equiv s$. Without loss of generality, we assume that $k \equiv r$. Since $\operatorname{rad} F \mathfrak{X}$ is $F J^{*}, \operatorname{rad} F X$ is one-dimensional. Therefore it follows that

$$
F X \cong P \oplus\left(n-m_{s}-2\right) S_{0} \oplus m_{s} S_{1} .
$$

In this case, the structure of $F X$ is completely determined by the parameters.

\subsection{Type (4) $F \mathfrak{X} \cong F[x] /\left(x^{3}\right)$}

We set that $M_{i} \cong F \mathfrak{X} /(\operatorname{rad} F \mathfrak{X})^{i}$ as $F \mathfrak{X}$-modules for $i=1,2,3$. We set $M=A_{1}-k A_{0}$. Then $\operatorname{rad}^{2} F \mathfrak{X}=F J^{*}$ and $\operatorname{rad} F \mathfrak{X}=F J^{*}+F M^{*}$.

We set $t=r k_{p}(M)=r k_{F}(M)$. Since $\left(M^{*}\right)^{2}=\mu J^{*} \neq 0,(F X) J^{*} \subset(F X) M^{*}$. Therefore it follows that

$$
F X \cong M_{3} \oplus(t-2) M_{2} \oplus(n-2 t+1) M_{1}
$$

Interesting examples are the association schemes defined by the $(25,12,5,6)$-strongly regular graphs. Their adjacency algebras over a field of characteristic 5 are type (4). There are 15 strongly regular graphs with parameters $(25,12,5,6)$, but the only one of them is selfcomplementary, so there are 8 non-isomorphic association schemes. Then the structures of the modular standard modules are as follows:

\begin{tabular}{lc}
\hline$F X$ & $\begin{array}{c}\text { The number of non-isomorphic } \\
\text { association schemes }\end{array}$ \\
\hline$M_{3} \oplus 10 M_{2} \oplus 2 M_{1}$ & 5 \\
$M_{3} \oplus 9 M_{2} \oplus 4 M_{1}$ & 2 \\
$M_{3} \oplus 7 M_{2} \oplus 8 M_{1}$ & 1 \\
\hline
\end{tabular}




\subsection{Type (5) $F \mathfrak{X} \cong F[x, y] /\left(x^{2}, x y, y^{2}\right)$}

In this case, $p \mid v_{1}$ and $p \mid v_{2}+1$, or $p \mid v_{1}+1$ and $p \mid v_{2}$. Let $\alpha$ and $\beta$ be $p \mid v_{\alpha}$ and $p \mid v_{\beta}+1$. In this case, we can define an isomorphism $F \mathfrak{X} \rightarrow F[x, y] /\left(x^{2}, x y, y^{2}\right)$ by $A_{\alpha} \mapsto x$ and $A_{0}+A_{\alpha}+A_{\beta} \mapsto y$. From here, we identify them.

Now we consider $\widehat{F X}$. Consider a basis $\left\{\tau_{0}, \tau_{\alpha}-\tau_{0}, \tau_{0}-\tau_{\beta}\right\}$ of $\widehat{F X}$. Then the matrix representation of $F \mathfrak{X}$ on $\widehat{F \mathfrak{X}}$ with respect to this basis is as follows:

$$
x \mapsto\left(\begin{array}{ccc}
0 & 0 & 0 \\
0 & 0 & 1 \\
0 & 0 & 0
\end{array}\right), \quad y \mapsto\left(\begin{array}{ccc}
0 & 0 & 1 \\
0 & 0 & 0 \\
0 & 0 & 0
\end{array}\right)
$$

Now we consider the structure of $F X$. Let

$$
F X=M_{1} \oplus M_{2} \oplus \cdots \oplus M_{r}
$$

be an indecomposable decomposition of $F X$. Since $\operatorname{dim}_{F} F X y=1$, there is the unique $M_{i}$ such that $M_{i} y \neq 0$. We assume $M_{1} y \neq 0$.

Since $F \mathfrak{X}$ has tame representation type, we can classify all indecomposable $F \mathfrak{X}$-modules [3, Section 4.3]. We have the following.

Proposition 13 Let $M$ be an indecomposable $F \mathfrak{X}$-module such that $\operatorname{dim}_{F} M y=0$. Then the corresponding representation is one of the following:

$$
\begin{aligned}
& \Lambda_{1}: x \mapsto(0), \quad y \mapsto(0) \text { (simple module), } \\
& \Lambda_{2}: x \mapsto\left(\begin{array}{ll}
0 & 1 \\
0 & 0
\end{array}\right), \quad y \mapsto\left(\begin{array}{ll}
0 & 0 \\
0 & 0
\end{array}\right) .
\end{aligned}
$$

Proposition 14 Let $M$ be an indecomposable $F \mathfrak{X}$-module such that $\operatorname{dim}_{F} M y=1$. Then the corresponding representation is one of the following:

$$
\begin{aligned}
\Gamma_{1}(\lambda): x \mapsto\left(\begin{array}{ll}
0 & 1 \\
0 & 0
\end{array}\right), \quad y \mapsto\left(\begin{array}{ll}
0 & \lambda \\
0 & 0
\end{array}\right)(\lambda \neq 0), \\
\Gamma_{2}: x \mapsto\left(\begin{array}{ll}
0 & 0 \\
0 & 0
\end{array}\right), \quad y \mapsto\left(\begin{array}{ll}
0 & 1 \\
0 & 0
\end{array}\right), \\
\Gamma_{3}: x \mapsto\left(\begin{array}{lll}
0 & 0 & 1 \\
0 & 0 & 0 \\
0 & 0 & 0
\end{array}\right), \quad y \mapsto\left(\begin{array}{lll}
0 & 0 & 0 \\
0 & 0 & 1 \\
0 & 0 & 0
\end{array}\right),
\end{aligned}
$$




$$
\begin{aligned}
& \Gamma_{4}: x \mapsto\left(\begin{array}{ccc}
0 & 1 & 0 \\
0 & 0 & 0 \\
0 & 0 & 0
\end{array}\right), y \mapsto\left(\begin{array}{lll}
0 & 0 & 1 \\
0 & 0 & 0 \\
0 & 0 & 0
\end{array}\right), \\
& \Gamma_{5}: x \mapsto\left(\begin{array}{llll}
0 & 0 & 1 & 0 \\
0 & 0 & 0 & 1 \\
0 & 0 & 0 & 0 \\
0 & 0 & 0 & 0
\end{array}\right), \quad y \mapsto\left(\begin{array}{llll}
0 & 0 & 0 & 1 \\
0 & 0 & 0 & 0 \\
0 & 0 & 0 & 0 \\
0 & 0 & 0 & 0
\end{array}\right) .
\end{aligned}
$$

We set $t=r k_{p}\left(A_{\alpha}\right)=r k_{F}\left(A_{\alpha}\right)$. From Propositions 5 and $13, M_{1}$ must be selfcontragradient. It follows that $F X \cong \Gamma_{5} \oplus(t-2) \Lambda_{2} \oplus(n-2 t) \Lambda_{1}, \Gamma_{1}(\lambda) \oplus$ $(t-1) \Lambda_{2} \oplus(n-2 t) \Lambda_{1}$, or $\Gamma_{2} \oplus t \Lambda_{2} \oplus(n-2 t-2) \Lambda_{1}$.

Let us denote the column space of $A_{\alpha}$ by $\left\langle A_{\alpha}\right\rangle$ and the all-one column vector by 1 . Then $(F X) J^{*} \subset(F X) A_{\alpha}$ if and only if $\mathbf{1} \in\left\langle A_{\alpha}\right\rangle$. We assume that $\mathbf{1} \in\left\langle A_{\alpha}\right\rangle$. Let us denote the $i$-th column vector of $A_{\alpha}$ by $A_{\alpha}(i)$. We set $\mathbf{1}=\sum_{i=1}^{n} a_{i} A_{\alpha}(i)$, where $a_{i} \in F$ for all $i$. Then it follows that

$$
F X \cong\left\{\begin{array}{lll}
\Gamma_{5} \oplus(t-2) \Lambda_{2} \oplus(n-2 t) \Lambda_{1} & \text { if } \mathbf{1} \in\left\langle A_{\alpha}\right\rangle \text { and } \sum_{i=1}^{n} a_{i} \equiv 0 \quad(\bmod p), \\
\Gamma_{1}(\lambda) \oplus(t-1) \Lambda_{2} \oplus(n-2 t) \Lambda_{1} & \text { if } \mathbf{1} \in\left\langle A_{\alpha}\right\rangle \text { and } \sum_{i=1}^{n} a_{i} \not \equiv 0 \quad(\bmod p), \\
\Gamma_{2} \oplus t \Lambda_{2} \oplus(n-2 t-2) \Lambda_{1} & \text { if } \mathbf{1} \notin\left\langle A_{\alpha}\right\rangle .
\end{array}\right.
$$

The example whose modular standard module contains $\Gamma_{5}$ is the association scheme defined by the $(16,5,0,2)$-strongly regular gaph. The adjacency algebra over a field of characteristic 2 is type (5) and $F X \cong \Gamma_{5} \oplus 4 \Lambda_{2} \oplus 4 \Lambda_{1}$.

The example whose modular standard module contains $\Gamma_{2}$ is the association scheme defined by the triangular graph $T(8)$. The adjacency algebra over a field of characteristic 2 is type (5) and $F X \cong \Gamma_{2} \oplus 6 \Lambda_{2} \oplus 14 \Lambda_{1}$. There are 4 strongly regular graphs with parameters $(28,12,6,4)$, namely $T(8)$ and three Chang graphs. We know that $T(8)$ is characterized by the 2-rank $r k_{2}\left(A_{1}\right)$ (See [5]), but $T(8)$ is also characterized by the structure of the modular standard module. Because it follows that $F X \cong \Gamma_{5} \oplus 6 \Lambda_{2} \oplus 12 \Lambda_{1}$ for the association scheme defined by a Chang graph.

The example whose modular standard module contains $\Gamma_{1}(\lambda)$ is the association scheme defined by the $(36,14,4,6)$-strongly regular graphs. The adjacency algebras over a field of characteristic 3 are type (5). There are 180 strongly regular graphs with parameters $(36,14,4,6)$. Two of them have the structure of the modular standard module $F X \cong \Gamma_{1}(\lambda) \oplus$ $12 \Lambda_{2} \oplus 10 \Lambda_{1}$.

Notes In the cases type (1) and (3), the structure of the modular standard module is determined completely by the multipicities (if the association scheme is defined by a strongly regular graph, they are determined by the parameters). Therefore we are interested in type 
(2), (4) and (5). In the cases type (2) and (4), we can determine the structure of the modular standard module by the $p$-rank of the corresponding matrix and the parameters of the corresponding strongly regular graph.

Here we focus on the case type (5). Then there is the possibility that we can obtain more information than the $p$-rank. Namely, they have the same $p$-rank of $A_{\alpha}$, but their structures of the modular standard module are non-isomorphic. Such an example is $(36,15,6,6)$-strongly regular graph. There are 227 strongly regular graphs with the parameter $(36,15,6,6)$ in the list by Spence [10], [11]. There are 60 strongly regular graphs such that $r k_{2}\left(A_{2}\right)=14$. Then three strongly regular graphs of them have $F X \cong \Gamma_{2} \oplus 14 \Lambda_{2} \oplus 6 \Lambda_{1}$, and the others $F X \cong \Gamma_{5} \oplus 12 \Lambda_{2} \oplus 8 \Lambda_{1}$. We do not know the examples that there are all of three cases with the same $p$-rank.

In general, it is not so easy to calculate the value $\lambda$ for $\Gamma_{1}(\lambda)$. We do not know the value $\lambda$ and its range.

\section{Acknowledgment}

The authors are thankful to the referee for lots of helpful remarks and suggestions.

\section{References}

1. Z. Arad, E. Fisman, and M. Muzychuk, "Generalized table algebras," Israel J. Math. 144 (1999), 29-60.

2. E. Bannai and T. Ito, "Algebraic combinatorics. I," Association Schemes, Benjamin-Cummings, Menlo Park, CA, 1984.

3. D. Benson, Representation and Cohomology I, Cambridge, 1995.

4. A.E. Brouwer, A.M. Cohen, and A. Neumaier, Distance-Regular Graphs, Springer-Verlag, Berlin, Heidelberg, 1989.

5. A.E. Brouwer and C.A. van Eijl, "On the p-rank of the adjacency matrices of strongly regular graphs," $J$. Algebraic Combin. 1 (1992), 329-346.

6. A. Hanaki, "Semisimplicity of adjacency algebras of association schemes," J. Algebra 225 (2000), 124-129.

7. H. Nagao and Y. Tsushima, Representations of Finite Groups, Academic Press, San Diego CA, 1987.

8. R. Peeters, "Uniqueness of strongly regular graphs having minimal p-rank," Linear Algebra Appl. 226-228 (1995), 9-31.

9. R. Peeters, "On the $p$-ranks of the adjacency matrices of distance-regular graphs," J. Algebraic Combin 15 (2002), 127-149.

10. E. Spence, "Regular two-graphs on 36 vertices," Linear Algebra Appl. 459-497 (1995), 226-228.

11. E. Spence, http://www.maths.gla.ac.uk/\%7ees/srgraphs.html .

12. P.-H. Zieschang, An Algebraic Approach to Association Schemes, vol. 1628, Lecture Notes in Math Springer, Berlin, Heidelberg, New York, 1996. 